\title{
Bone material strength is associated with areal BMD but not with prevalent fractures in older women
}

\author{
R. Rudäng ${ }^{1}$ - M. Zoulakis ${ }^{1}$ - D. Sundh ${ }^{1}$ - H. Brisby ${ }^{2}$ A. Diez-Perez ${ }^{3}$ L. Johansson ${ }^{1}$ \\ D. Mellström ${ }^{1} \cdot$ A. Darelid ${ }^{1} \cdot$ M. Lorentzon ${ }^{1}$
}

Received: 25 June 2015 / Accepted: 10 November 2015 /Published online: 2 December 2015

(C) The Author(s) 2015. This article is published with open access at Springerlink.com

\begin{abstract}
Summary Reference point indentation is a novel method to assess bone material strength index (BMSi) in vivo. We found that BMSi at the mid-tibia was weakly associated with spine and hip areal bone mineral density but not with prevalent fracture in a population-based cohort of 211 older women.

Introduction Reference point indentation is a novel method to assess BMSi in vivo. Lower BMSi has been observed in patients with prior fracture than in controls, but no association between BMSi and areal bone mineral density (aBMD) has been found. Population-based association studies and prospective studies with BMSi and fractures are lacking. We hypothesized that BMSi would be associated with prevalent fractures in older Swedish women. The aim was to investigate the associations between $\mathrm{BMSi}$, aBMD, and prevalent fracture in older women. Methods Two hundred eleven women, mean age $78.3 \pm$ 1.1 years, were included in this cross-sectional, populationbased study. BMSi was assessed using the OsteoProbe device at the mid-tibia. Areal BMD of the hip, spine, and nondominant radius was measured using dual-energy X-ray absorptiometry (DXA). Fracture history was retrieved using
\end{abstract}

M. Lorentzon

mattias.lorentzon@medic.gu.se

1 Geriatric Medicine, Department of Internal Medicine and Clinical Nutrition, Institute of Medicine, The Sahlgrenska Academy, Sahlgrenska University Hospital, Building K, 6th Floor, 431 80 Mölndal, Sweden

2 Department of Orthopaedics, Sahlgrenska University Hospital, University of Gothenburg, Gothenburg, Sweden

3 Department of Internal Medicine, Institut Mar Investigació Mèdica (IMIM), Autonomous University of Barcelona, Barcelona, Spain questionnaires, and vertebral fractures were identified using vertebral fracture assessment (VFA) by DXA.

Results One hundred ninety-eight previous fractures in 109 subjects were reported. A total of 106 women had a vertebral fracture, of which 58 women had moderate or severe fractures. An inverse correlation between BMSi and weight $(r=-0.14, p=$ $0.04)$ was seen, and BMSi differed according to operator (ANOVA $p<0.01$ ). Adjusting for weight and operator in a linear regression model, we found that $\mathrm{BMSi}$ was positively associated with aBMD of the total hip $(\beta=0.14, p=0.04)$, non-dominant radius $(\beta=0.17, p=0.02)$, and lumbar spine (L1-L4) $(\beta=0.14$, $p<0.05)$. Using logistic regression, we could not find any association in crude or adjusted BMSi (for age, weight, height, walking speed, calcium intake, smoking, bisphosphonate and glucocorticoid use, and operator) with prevalent fractures.

Conclusion We conclude that BMSi is associated with aBMD but not with prevalent fracture in a population-based cohort of 211 older women.

Keywords Bone material strength · Bone mineral density · Fracture

\section{Introduction}

Osteoporotic fractures constitute a major public health problem in Sweden, in which every second women and fifth man after age 50 years will suffer from such a fracture [1]. Bone mineral density (BMD), as derived by dual-energy X-ray absorptiometry (DXA), is a well-studied predictor of fracture risk and used as the golden standard to diagnose osteoporosis and determine fracture risk today [2]. Osteoporosis, defined as $\mathrm{BMD} \leq-2.5 \mathrm{SD}$ below the mean value of a young adult population, does however not alone explain fracture risk, since many fractures occur in patients with normal BMD and not all patients with low bone mass do 
fracture [3]. Other clinical risk factors, such as advanced age, prior fracture, heredity, smoking, and medications such as oral glucocorticoids, contribute independently to fracture risk [4, 5]. Also, geometrical and microarchitectural properties as well as more sophisticated assessment of bone strength by finite element analyses are associated with fractures, partially independent of BMD [6, 7]. Recently, much interest has been given material strength properties of the bone, as measured with non-imaging techniques. Reference point indentation (RPI) is a novel such method for assessment of bone material strength index (BMSi) in vivo [8]. The method includes a device with a needle being pushed with a defined force on the bone surface, and the outcome measure is the distance the needle is pushed into the bone or indentation distance increase (IDI). IDI determined with one RPI instrument, the BioDent (ActiveLife Tech, Inc., CA, USA), has been shown to be able to discriminate between controls without fracture and cases with either osteoporotic fractures [8], osteoporotic hip fractures, or atypical hip fractures [9]. The BioDent and other previous RPI devices included a reference probe and required displacement of the periosteum before the indentation procedure. The most recently introduced RPI device, the OsteoProbe (Active Life Scientific Inc., CA, USA), requires no reference probe and can be used through the soft tissue and periosteum, which makes it easy to operate and does not require extensive training according to the manufacturer $[10,11]$. The OsteoProbe measures BMSi, which is a unitless parameter, derived as the ratio between the average IDI (of repeated measurements) in a reference standard of polymethylmetacrylate (PMMA) and in the bone. So far, only one study has investigated the associations between prevalent fractures and BMSi derived by the OsteoProbe. In a study on 90 men and women (40.4-85.5 years) with low bone mass, Malgo et al. demonstrated an inverse association between BMSi and prevalent fragility fractures, independent of areal BMD [12]. The microindentation-derived BMSi is believed to describe a bone component different from BMD, and other studies using the OsteoProbe have not reported any association with DXA-derived areal BMD (aBMD). Farr et al. showed that diabetic subjects had approximately $10 \%$ lower BMSi than age-matched controls, also after adjustment for BMI, while aBMD did not differ between the groups after BMI adjustment [13]. Furthermore, a study comparing 42 Norwegian and 46 Spanish women demonstrated lower BMSi and higher BMD in the Norwegian women as compared to the Spanish women [14].

The aim of this study was to investigate the associations between BMSi and prevalent fractures, including vertebral fractures (defined by vertebral fracture assessment (VFA), with DXA), in a population-based cohort of 211 women between 75 and 80 years of age. Furthermore, we sought to examine possible associations between BMSi and DXA-derived aBMD in the same cohort.

\section{Materials and methods}

A population-based study of older women, 75 and 80 years of age, was initiated in 2013 with the aim to determine predictors of hip and other fractures. Women were identified using national population registers, contacted by mail and telephone, and asked to participate in the study. Women had to be able to understand Swedish and walk (with or without an aid) to be eligible for the study. From September 2014 to March 2015, all participating women $(n=496)$ were considered for participation in the present study. Of these, a total of 482 subjects were contacted by telephone and asked to participate in the BMSi exam. One study participant declined any further studies at first visit, one had extensive osteosynthesis material of the lower limbs, three subjects could not be reached, seven had leg ulcers, and two were under cytostatic treatment and currently immunodeficient and were therefore excluded. Of the contacted subjects, 218 agreed to participate in the study, corresponding to $45.2 \%$ of the initially contacted. Written and oral consent was obtained from all study participants. The Regional Ethical Review Board at the University of Gothenburg approved the study.

Height and weight were measured using standardized equipment. As a measure of current physical fitness, the subjects were instructed to walk a $10-\mathrm{m}$ distance in their selfchosen pace. This was conducted twice, and the mean time was used to calculate walking speed $(\mathrm{m} / \mathrm{s})$. Calcium intake was estimated from reported daily intake of dairy products including contingent medical calcium supplementation and expressed in $\mathrm{mg} /$ day.

\section{Bone mineral density}

$\operatorname{aBMD}\left(\mathrm{g} / \mathrm{cm}^{2}\right)$ of the total hip, femur neck, lumbar spine, and the mid-third of the non-dominant radius were assessed using DXA (Discovery A, Hologic). aBMD of the lumbar spine was calculated as the mean of L1-L4, excluding fractured vertebrae. $T$ scores were generated by the DXA software (version 13.1.0) using the manufacturer-specific database. The coefficient of variation (CV) for aBMD of the total hip was $0.83 \%$, femur neck $1.3 \%$, lumbar spine $0.68 \%$, and radius $3.1 \%$. $\mathrm{CVs}$ for the spine and hip (including neck) measurements were obtained by duplicate measurements on 30 women, 75-80 years old, while the radius $\mathrm{CV}$ was obtained using three measurements on one woman. One operator performed all measurements.

\section{Bone material strength}

BMSi was assessed using the OsteoProbe ${ }^{\circledR}$ (Active Life Scientific Inc., CA, USA), which is a RPI device developed for microindentation in vivo $[10,11]$. The OsteoProbe ${ }^{\circledR}$ is a handheld instrument with a needle of stainless steel with a trigger 
mechanism, allowing testing on the anterior face of the midtibia under local anesthesia $(2 \mathrm{ml}$, mepivacaine, $10 \mathrm{mg} / \mathrm{ml})$. The needle is applied through the skin and firmly pushed perpendicular to the bone surface (Fig. 1). When the load/ force reaches $10 \mathrm{~N}$, a trigger mechanism will initiate an impact where the needle will be pushed into the bone surface with a force of $30 \mathrm{~N}$ during less than a millisecond. This will create an indentation, or microfracture, on the bone surface. The software registers the distance from the needle tip right before impact and right after, a distance called the IDI. Directly after a number of repeated measurements separated from one another by at least $2 \mathrm{~mm}$, five measurements are performed on a piece of PMMA. The BMSi is calculated as 100 times the ratio of the mean IDI in the PMMA and the tibia.

In the present study, at least 11 valid indentations were performed on each subject, of which the first indentation (perforating the skin) was discarded since the probe is not established on the bone and the indentation value is affected by amount and toughness of soft tissue and skin. All other indentations were checked for validity, and indentations that for obvious technical reasons failed were deleted. Of the 218 subjects who agreed to participate in the study, a total of 7 subjects had to be excluded, due to failed indentations. Out of these seven subjects, six of them were excluded because of difficulties acquiring enough number of indentations secondarily to firm or large amount of subcutaneous tissue or due to marked irregularities on the tibial surface. In one subject, the measurement was cancelled before it had started because of discomfort for the patient during the application of local anesthesia. Thus, in all, 211 subjects were included in the subsequent analyses.

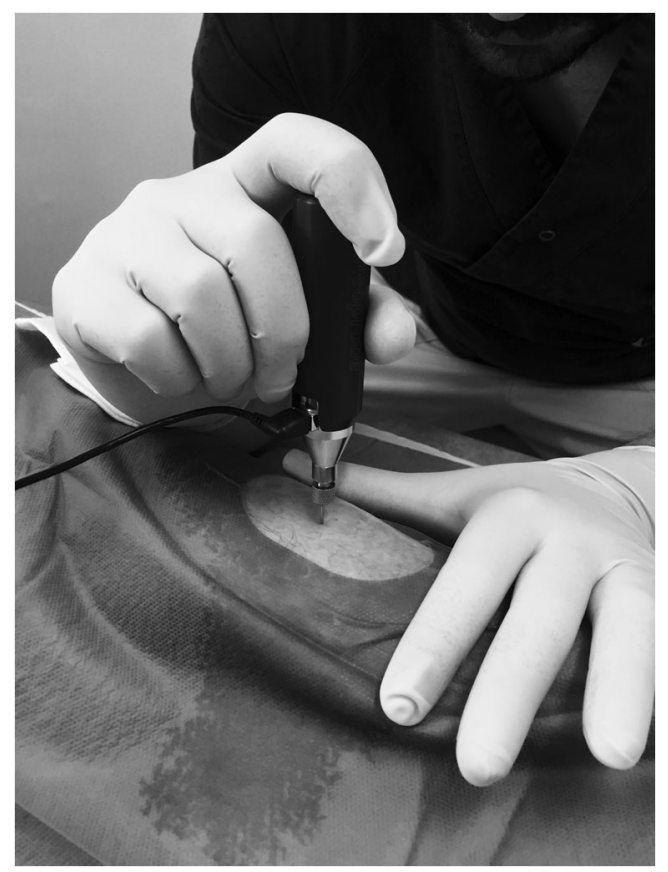

Fig. 1 Performing microindentations using the OsteoProbe
The measurements were performed by four operators, and in the first 100 measurements, there were at least two operators present, to assure that all operators performed the procedure uniformly. The first 100 subjects were contacted by telephone 3 days after the procedure, and no adverse events were reported.

The intraobserver $\mathrm{CV}$ for the method was $3.2 \%$, which was calculated by measuring 30 subjects twice. Both measurements were made by the same operator, on the same leg with an approximately $2-\mathrm{cm}$ distance between the two measurement sites, including a short break between the measurements where the indentation needle was exchanged for a new needle. The reported CV corresponds to the mean of the CVs of each of the 30 paired measurements, calculated as 100 times the ratio between the $\mathrm{SD}$ and the mean. Interobserver $\mathrm{CV}$ was $5.2 \%$, which was calculated measuring another set of 30 subjects in the same manner as previously described but with two different operators performing the first and second measurement. All four operators participated in the assessments of both CVs. Each subject was measured by one operator, except for in 30 subjects who were measured by two different operators in order to calculate interobserver $\mathrm{CV}$.

After analyzing all BMSi measurements, we found that the means were significantly different between the four operators (operator $1(n=36) 75.7 \pm 7.8($ mean $\pm \mathrm{SD})$, operator $2(n=41)$ $75.3 \pm 8.1$, operator $3(n=60) 79.4 \pm 7.0$, and operator $4(n=74)$ $72.6 \pm 6.3$, ANOVA $p<0.001)$. To adjust for operator in the present study, we used dummy variables as independent variables in the linear and logistic regression models.

\section{Fracture history}

Fracture history was retrieved by a questionnaire, and subjects were asked to report fracture site and their age at the time of the fracture. Peripheral fractures were defined as fractures of the limbs (excluding feet and hands), i.e., radius, ulna, humerus, tibia, fibula, and the femur. Osteoporotic fractures were defined by skeletal site as fractures of the distal forearm, proximal humerus, vertebral fractures, hip fractures, and pelvic fractures. All reported fractures after the age of 50 years were included in the analyses. Thus, the control group in Table 2 (no fracture) refers to no fractures after the age of 50 years. All fractures, irrespective of trauma type, were included.

Vertebral fractures were identified using VFA by DXA [15], where fractures were classified according to the semiquantitative method of Genant [16]. A lateral scan of the DXA was used to visually detect morphologic deformities of the vertebrae, and by measurement of the anterior, middle, and posterior aspects of the vertebrae, detected fractures were graded due to their height reduction as mild (20-25\%), moderate (25-40\%), or severe ( $>40 \%)$. The morphology of adjacent vertebrae was also taken into account when diagnosing vertebral compressions. The same physician evaluated all 
lateral DXA scans. In the present study, only moderate and severe vertebral fractures were accounted for as prevalent fractures whereas subjects with only mild fractures were excluded from further analysis. The operator's reproducibility was tested on 550 vertebras. For vertebras T4-L4, the intraobserver agreement was $98.9 \%$ (kappa score 0.85 ) and the interobserver agreement was $97.6 \%$ (kappa score 0.72 ). When mild vertebral fractures were excluded, the intraobserver agreement was $100 \%$ (kappa score 1.0) and the interobserver agreement was $99.6 \%$ (kappa score 0.95 ).

\section{Statistical analyses}

Correlations between BMSi and anthropometrics, environmental factors, and DXA measurements were investigated by Pearson correlation test. Associations between aBMD variables and BMSi were evaluated with linear regression models including weight and dummy variables for operator as independent variables in the first analysis and also including all other covariates in the second analysis. Evaluation of BMSi in relation to smoking, bisphosphonate and glucocorticoid use, and fracture history was made by independent samples $t$ test. In the latter analysis, weight and operator-adjusted BMSi was used, which was calculated using linear regression, where the unstandardized residuals were added to the mean value to achieve adjusted values of BMSi. Logistic regression models, including all covariates, were used to determine whether BMSi was associated with fracture or not. Weight was not normally distributed, as observed also in other populations $[17,18]$ and therefore log-transformed before inclusion in the statistical models. $P$ values less than 0.05 were considered significant. All analyses were performed using SPSS (Version 20, SPSS, Inc., Chicago, IL, USA).

\section{Results}

A total of 211 subjects were included in the present study. The mean value of the BMSi measurements was $75.6 \pm 7.6$ with a range from 52.9 to 93.3. Other characteristics of the subjects, including age, anthropometrics, environmental factors, and aBMD, are presented in Table 1.

\section{Correlations between BMSi, anthropometrics, aBMD, and medication use}

Crude BMSi was inversely correlated to weight, but no correlations were seen with age, height, walking speed, calcium intake, or any of the aBMD measurements (Table 1). There were no significant differences in BMSi between smokers and non-smokers, or ever smokers and never smokers, respectively. No differences were seen between current users of bisphosphonates or glucocorticoids and non-users, respectively, but ever users of bisphosphonates had slightly higher BMSi (although only borderline significant) than never users, which was not the case for ever glucocorticoid-treated subjects (Table 2). Using BMSi adjusted for weight and operator did not significantly alter these results, except for the difference between ever and never users of bisphosphonates, which was no longer borderline significant.

\section{BMSi and the association with aBMD}

Using a linear regression model with aBMD as dependent variable and crude BMSi, weight, and operator dummy variables as independent variables, we found that BMSi was positively associated with aBMD of the total hip, femur neck, lumbar spine, and the radius (Table 1). After also including age, height, walking speed, calcium intake, smoking status, and use of bisphosphonates and glucocorticoids as covariates, only aBMD of the radius remained significant, whereas aBMD of the hip and lumbar spine were of borderline significance (Table 1).

\section{BMSi and the absence of association with prevalent fractures}

A total of 198 fractures in 109 subjects were reported in the questionnaire. Of these, there were 86 subjects with fractures that occurred after the age of 50. Using VFA by DXA, we found a total of 176 deformed vertebrae in 106 subjects, interpreted as prevalent vertebral fractures, of which 91 were mild, 57 moderate, and 28 severe. A total of 58 women had moderate and/or severe vertebral fractures, whereas 48 subjects only had mild fractures. Of these 48 subjects, a total of 31 had no other reported fractures and were therefore excluded from further analyses. Including all reported fractures after the age of 50 and all moderate and severe VFA-verified vertebral fractures, there were 117 women who were assigned to the fracture group and 63 subjects with no reported fractures or no significant vertebral deformities were used as controls in the following analyses.

There were no differences in either crude or weight and operator-adjusted BMSi between the fracture group and the controls, but aBMD at the hip was significantly lower in the former group (Table 3). Women with VFA-verified vertebral fractures $(n=58)$ had lower aBMD of the hip than the controls, but no difference was seen in BMSi. Reported peripheral $(n=53)$ and osteoporotic $(n=89)$ fractures occurring after the age of 50 showed a similar pattern with no differences in BMSi but lower $\mathrm{aBMD}$ at the hip and for peripheral fractures also at the lumbar spine. Subjects with peripheral fractures after the age of $50 \mathrm{had}$ slightly higher calcium intake than the controls (Table 3).

Including current use of glucocorticoids and bisphosphonates, smoking, age, height, weight, calcium intake, operator, and walking speed as independent variables together with BMSi in a logistic regression model, we found no 
Table 1 Characteristics of the study subjects and BMSi associations

Subjects $(n=211) \quad$ BMSi $r \quad p \quad \underset{\beta}{\text { Weight and operator-adjusted BMSi }} \quad p \quad \underset{\beta}{\text { Fully adjusted BMSi }} p$

$\beta$

\begin{tabular}{|c|c|c|c|c|c|c|c|}
\hline Age (years) & $78.3 \pm 1.1$ & -0.07 & 0.35 & & & & \\
\hline Weight $(\mathrm{kg})$ & $69.1 \pm 12.6$ & -0.14 & 0.04 & & & & \\
\hline Height $(\mathrm{cm})$ & $161.1 \pm 5.8$ & 0.04 & 0.56 & & & & \\
\hline Walking speed $(\mathrm{m} / \mathrm{s})$ & $2.0 \pm 0.4$ & 0.04 & 0.59 & & & & \\
\hline Calcium intake (mg/day) & $743 \pm 407$ & 0.03 & 0.67 & & & & \\
\hline Total hip aBMD $\left(\mathrm{g} / \mathrm{cm}^{2}\right)$ & $0.796 \pm 0.112$ & 0.05 & 0.51 & 0.14 & 0.04 & 0.13 & 0.06 \\
\hline Femur neck aBMD $\left(\mathrm{g} / \mathrm{cm}^{2}\right)$ & $0.652 \pm 0.100$ & 0.05 & 0.45 & 0.13 & $<0.05$ & 0.13 & 0.06 \\
\hline Lumbar spine aBMD $\left(\mathrm{g} / \mathrm{cm}^{2}\right)$ & $0.934 \pm 0.161$ & 0.06 & 0.36 & 0.14 & $<0.05$ & 0.13 & 0.06 \\
\hline Radius aBMD $\left(\mathrm{g} / \mathrm{cm}^{2}\right)$ & $0.575 \pm 0.078$ & 0.08 & 0.22 & 0.17 & 0.02 & 0.16 & 0.03 \\
\hline
\end{tabular}

Mean values and standard deviations are shown in the first column, and the second column displays $r$-coefficients calculated using Pearson correlation. Unstandardized $\beta$ were calculated using linear regression models with BMS as predictor, including weight and operator as independent variables in the fourth column. The fully adjusted model also included age, height, walking speed, calcium intake, current smoking, and current use of bisphosphonates and oral glucocorticoids as covariates

associations between BMSi and any prevalent fracture, vertebral fractures, peripheral fractures, or osteoporotic fractures (Table 4). Including also ever users of glucocorticoids and bisphosphonates did not change the outcome of these analyses (data not shown). On the other hand, areal BMD of the hip showed strong associations with all subgroups of fractures, which was also the case for lumbar spine aBMD regarding peripheral fractures (Table 4).

\section{BMSi in osteopenic/osteoporotic subjects}

Osteopenic/osteoporotic subjects (femur neck $T$ score $\leq-1, n=$ 176) had no different BMSi than subjects with normal aBMD $(n=35)(75.6 \pm 7.6$ vs. $75.5 \pm 7.6, p=0.93)$ nor were there any significant differences in weight or operator-adjusted BMSi between osteopenic/osteoporotic subjects and subjects with normal aBMD $(75.4 \pm 7.0$ vs. $76.6 \pm 6.8, p=0.36)$.

We investigated the 176 subjects with osteopenia/ osteoporosis according to fracture history status. A total of 101 subjects had reported fractures after age 50 and/or moderate and/or severe VFA-verified vertebral fractures whereas 46 subjects had no fractures. Thus, a total of 29 subjects with only mild vertebral fractures were excluded from the analyses. Using independent samples $t$ test, there were no differences between fractured subjects and controls $(n=46)$ in crude
BMSi (76.5 \pm 7.4 vs. $75.3 \pm 8.0, p=0.40)$ or operator and weight-adjusted BMSi (76.1 \pm 6.9 vs. 75.1 $\pm 7.0, p=0.43)$. Fractured women had lower aBMD of the total hip $(0.748 \pm$ 0.094 vs. $0.799 \pm 0.080, p=0.002)$ and the femur neck $(0.608 \pm$ 0.076 vs. $0.647 \pm 0.056, p=0.003$ ) than controls. Including all covariates in a logistic regression model (as above), there were no associations between BMSi and any fracture (odds ratio (OR) 0.85 (0.57-1.26)), vertebral fractures (OR 0.60 (0.35$1.02)$ ), peripheral fractures (OR $1.04(0.62-1.74))$, or osteoporotic fractures (OR $0.71(0.46-1.11))$ but strong associations between aBMD of the total hip and femur neck for all fractures (OR 2.42 (1.38-4.26) and OR 2.76 (1.42-5.33), respectively), vertebral fractures (OR 2.93 (1.41-6.11) and OR 3.48 (1.48-8.20), respectively), peripheral fractures (OR 5.63 (2.30-13.75) and OR 4.18 (1.82-9.59), respectively), and osteoporotic fractures (OR 2.97 (1.52-5.80) and OR 2.79 (1.335.82), respectively).

\section{Discussion}

In a population-based cohort of 211 women between 75 and 80 years of age, we could not demonstrate any difference in BMSi between fractured and non-fractured subjects, but a weak association between BMSi and aBMD was found. This

Table 2 BMSi according to smoking status, use of bisphosphonates, or p.o. glucocorticoids

\begin{tabular}{|c|c|c|c|c|c|c|}
\hline & $\begin{array}{l}\text { BMSi } \\
\text { Current user }\end{array}$ & $\begin{array}{l}\text { BMSi } \\
\text { Non-user }\end{array}$ & $p$ & $\begin{array}{l}\text { BMSi } \\
\text { Ever user }\end{array}$ & $\begin{array}{l}\text { BMSi } \\
\text { Never user }\end{array}$ & $p$ \\
\hline Smoking & $75.7 \pm 8.9(n=18)$ & $75.6 \pm 7.5(n=193)$ & 0.95 & $75.0 \pm 8.0(n=89)$ & $76.1 \pm 7.3(n=122)$ & 0.34 \\
\hline Bisphosphonates & $77.1 \pm 5.7(n=18)$ & $75.5 \pm 7.7(n=193)$ & 0.28 & $77.8 \pm 7.7(n=40)$ & $75.1 \pm 7.5(n=171)$ & 0.05 \\
\hline Glucocorticoids (p.o.) & $81.7 \pm 7.5(n=5)$ & $75.5 \pm 7.5(n=206)$ & 0.16 & $78.0 \pm 7.8(n=20)$ & $75.4 \pm 7.5(n=191)$ & 0.17 \\
\hline
\end{tabular}

Means and standard deviations are shown. Differences between groups evaluated using independent samples $t$ test 
Table 3 Associations between anthropometric variables, aBMD, and BMSi

\begin{tabular}{|c|c|c|c|c|c|}
\hline & $\begin{array}{l}\text { No fracture } \\
n=63\end{array}$ & $\begin{array}{l}\text { All fractures } \\
n=117\end{array}$ & $\begin{array}{l}\text { Vertebral fractures } \\
n=58\end{array}$ & $\begin{array}{l}\text { Peripheral fractures } \\
n=53\end{array}$ & $\begin{array}{l}\text { Osteoporotic fractures } \\
n=89\end{array}$ \\
\hline BMSi & $75.7 \pm 7.9$ & $76.1 \pm 7.4$ & $77.1 \pm 7.5$ & $75.5 \pm 7.0$ & $76.7 \pm 7.3$ \\
\hline $\mathrm{BMSi}$, adjusted for weight and operator & $75.7 \pm 7.1$ & $76.0 \pm 6.9$ & $76.9 \pm 6.9$ & $75.6 \pm 6.7$ & $76.7 \pm 6.8$ \\
\hline Age (years) & $78.2 \pm 1.1$ & $78.3 \pm 1.1$ & $78.2 \pm 1.1$ & $78.4 \pm 1.0$ & $78.3 \pm 1.1$ \\
\hline Weight (kg) & $70.2 \pm 13.4$ & $68.6 \pm 12.9$ & $66.9 \pm 13.2$ & $68.7 \pm 12.8$ & $68.3 \pm 13.2$ \\
\hline Height $(\mathrm{cm})$ & $162.1 \pm 5.7$ & $160.6 \pm 6.2$ & $160.3 \pm 6.0$ & $160.5 \pm 6.1$ & $160.2 \pm 6.4$ \\
\hline Walking speed $(\mathrm{m} / \mathrm{s})$ & $2.0 \pm 0.4$ & $2.0 \pm 0.4$ & $2.0 \pm 0.4$ & $2.0 \pm 0.5$ & $2.0 \pm 0.4$ \\
\hline Calcium intake (mg/day) & $682 \pm 362$ & $773 \pm 443$ & $783 \pm 465$ & $861 \pm 454 *$ & $813 \pm 461$ \\
\hline Total hip aBMD $\left(\mathrm{g} / \mathrm{cm}^{2}\right)$ & $0.843 \pm 0.109$ & $0.773 \pm 0.114^{* * *}$ & $0.765 \pm 0.112^{* * *}$ & $0.751 \pm 0.123^{* * *}$ & $0.770 \pm 0.114^{* * *}$ \\
\hline Femur neck aBMD $\left(\mathrm{g} / \mathrm{cm}^{2}\right)$ & $0.691 \pm 0.094$ & $0.638 \pm 0.104 * * *$ & $0.636 \pm 0.106^{* *}$ & $0.627 \pm 0.120^{* *}$ & $0.639 \pm 0.104 * *$ \\
\hline Lumbar spine aBMD $\left(\mathrm{g} / \mathrm{cm}^{2}\right)$ & $0.960 \pm 0.148$ & $0.912 \pm 0.167$ & $0.917 \pm 0.171$ & $0.881 \pm 0.153^{* *}$ & $0.915 \pm 0.171$ \\
\hline Radius aBMD (g/cm²) & $0.581 \pm 0.085$ & $0.571 \pm 0.075$ & $0.572 \pm 0.081$ & $0.554 \pm 0.078$ & $0.570 \pm 0.077$ \\
\hline
\end{tabular}

Means and standard deviations are shown. Differences between the values of the first column and the remaining columns were investigated using independent samples $t$ test. All fracture cases represent fractures after the age of 50, except VFA-verified vertebral fractures, which were not datable. Vertebral fractures refer to moderate and severe fractures verified by VFA. Cases with only mild vertebral fractures were excluded

${ }^{*} p<0.05, * * p<0.01, * * * p<0.001$

is in contrast to the only previously published study on prevalent fractures using the OsteoProbe, where Malgo et al. reported that BMSi was associated with prevalent fragility fracture in men and women with osteopenia or osteoporosis [12]. As a subanalysis, we also investigated the osteopenic/ osteoporotic women of our cohort, but we could not reveal any reduction in BMSi in regard to prevalent fracture. In contrast, also Diez-Perez found significant differences in microindentation values between hip fracture and nonfracture cases although with a different device and in a cyclic loading challenge testing [8].

We report an association between weight and BMSi, and when adjusting for weight, BMSi was positively associated with aBMD at the hip, spine, and radius. In contrast, Malgo et al. found no correlations between BMSi and aBMD [12]. Sosa et al. demonstrated that Norwegian women had higher aBMD but lower BMSi than Spanish women [14], while no association was seen between aBMD and BMSi. In the present study, the cohort of women was quite homogenous with a narrower age span but had a higher mean age than was the case in the examined populations in the previously performed studies. Given that the function of the mineral is to strengthen the collagen composite and that the actual bone strength is highly correlated with DXA-derived aBMD, it seems plausible that BMSi should in some way be correlated with aBMD [19].

It has not yet been well characterized what BMSi actually measures, in terms of bone material properties. We speculate that the cortical bone would be deteriorated, with a high agedependent cortical porosity in our cohort of older women, which would then be expected to negatively influence BMSi in the cortical bone. Therefore, peripheral fractures, to which cortical bone probably contributes the most, were also investigated in relation to BMSi. Surprisingly, there was no

Table 4 Fracture history is associated with aBMD but not BMSi

\begin{tabular}{lllll}
\hline & $\begin{array}{l}\text { All fractures } \\
n=117 \\
\text { OR (CI) }\end{array}$ & $\begin{array}{l}\text { Vertebral fractures } \\
n=58 \\
\text { OR (CI) }\end{array}$ & $\begin{array}{l}\text { Peripheral fractures } \\
n=53 \\
\text { OR (CI) }\end{array}$ & $\begin{array}{l}\text { Osteoporotic fractures } \\
n=89 \\
\text { OR (CI) }\end{array}$ \\
\hline BMSi & $0.89(0.63-1.27)$ & $0.67(0.43-1.05)$ & $0.96(0.61-1.50)$ & $0.74(0.51-1.09)$ \\
Total hip aBMD & $1.98(1.34-2.93)$ & $1.95(1.20-3.17)$ & $2.71(1.57-4.67)$ & $1.92(1.26-2.93)$ \\
Femur neck aBMD & $1.73(1.19-2.51)$ & $1.60(1.00-2.55)$ & $1.79(1.12-2.87)$ & $1.61(1.06-2.43)$ \\
Lumbar spine aBMD & $1.30(0.92-1.84)$ & $1.15(0.75-1.76)$ & $1.87(1.13-3.09)$ & $1.22(0.84-1.77)$ \\
Radius aBMD & $1.06(0.74-1.51)$ & $0.88(0.57-1.36)$ & $1.41(0.89-2.21)$ & $0.98(0.66-1.46)$ \\
\hline
\end{tabular}

Results presented as odds ratios (OR) and confidence intervals $(\mathrm{CI})$ for prevalent fracture per standard deviation decrease in BMSi and aBMD. Associations were tested using logistic regression, including operator (only for BMSi), age, weight, height, walking speed, calcium intake, current smoking, and current use of bisphosphonates and oral glucocorticoids. All fracture cases represent fractures after the age of 50, except VFA-verified vertebral fractures, which were not datable. Vertebral fractures refer to moderate and severe fractures verified by VFA. Cases with only mild vertebral fractures were excluded 
tendency of any association between BMSi and these fractures, indicating that BMSi does not reflect bone fragility in our particular study population. In some clinical situations, bone material properties may play a more predominant role in decreasing bone strength, like younger individuals with fragility fractures [12], diabetes [20], or incident use of glucocorticoids [21]; BMSi testing could perhaps be of greater value.

A strength of the present study is that we have investigated the associations between BMSi, prevalent fractures, and aBMD in the largest sample of women so far. In contrast to previous studies, our sample was population-based and not selected due to an event such as a recent prior fracture. It could be argued that using a larger, more homogenous and population-based sample, the risk of bias due to confounders affecting the BMSi associations would be smaller than in case-control studies.

There are some limitations in this study. Firstly, there were four different operators conducting the microindentation testing. To minimize the possible negative effect of the outcome, all operators trained together before starting the study, and in the first 100 subjects, at least two operators were present during the measurement of which one was observing and commenting on the technique if necessary. We also found that the mean BMSi for each operator differed which could not only be due to differences in the characteristics of the women measured but also due to differently performed measurements. To ensure that any possible bias introduced by operator and the measuring technique would be accounted for, we adjusted for operator using dummy variables in the regression models used although some potential effect on the results cannot completely be ruled out. Another weakness is that we have not been able to verify the self-reported fractures, and other previous studies have reported a significant discrepancy between reported and X-ray-verified fractures $[22,23]$. Furthermore, our used definition of osteoporotic fractures was based only on skeletal site, and we included all reported fractures, irrespective of trauma type, which could have attenuated possible associations. However, we also included VFA-verified vertebral fractures, and in the separate analysis on these subjects, no association between fractures and BMSi was seen. Arguing against errors introduced by fracture misclassification (i.e., fractures being self-reported), we found clear associations with aBMD, indicating that also the self-reported fractures were indeed due to presence of skeletal fragility. We lacked information concerning the nature of the trauma causing the fracture, which may infer that several of the reported fractures are actually caused by moderate or even severe trauma. There are, however, studies reporting that also fractures caused by moderate and severe trauma are associated to bone fragility in a similar way as low-energy fractures are [24,
25]. We tested association between BMSi and several aBMD sites, introducing uncertainty of multiple testing, although the aBMD variables were intercorrelated (femoral neck vs. total hip BMD $(r=0.83)$ and radius aBMD vs. total hip $(r=0.65))$. The weak associations found between BMSi and aBMD would not remain significant if adjustments were made for multiple comparisons.

Since we are unable to corroborate the previously published reports finding associations between BMSi and prevalent fracture, we performed a very extensive evaluation of our BMSi protocol and analysis. We performed as much as 30 dual measurements, using more indentations than other investigators, and could demonstrate a low intraobserver CV of $3.2 \%$. Farr et al., however, reports an even lower intraobserver $\mathrm{CV}$ (precision error) of $1.65 \%$, calculated on ten subjects [13], while Malgo reports a $\mathrm{CV}$ of $<10 \%$, without presenting the method of calculation [12]. In the study comparing Norwegian and Spanish women, a CV of $9.1 \%$ including 16 subjects was reported [14]. We also thoroughly investigated the interobserver $\mathrm{CV}$ found to be $5.2 \%$, which is still low in comparison to other investigators. Thus, our rather low CVs obtained with high standards support that we have conducted the method accurately and that our observations are likely not the results of insufficiently performed microindentations.

The number of moderate and severe vertebral fractures was high in the present study, corresponding to a prevalence of $27.5 \%$ in the studied cohort, which is in line with high prevalence numbers previously reported for older Swedish women [26], who have among the highest fracture risk in the world $[27,28]$.

We conclude that in a population-based cohort of older Swedish women, there were no associations between prevalent fracture and BMSi. We also demonstrate a weak association between weight-adjusted BMSi and aBMD of relevant skeletal sites. As a result of our study not being able to corroborate a previous study that has found associations between prevalent fractures and $\mathrm{BMSi}$, we believe that further studies are needed to establish if BMSi can aid in characterizing skeletal fragility and improve fracture prediction, in particular in older populations.

Acknowledgments This study was funded by the Swedish Research Council (VR), the Lundberg Foundation, Gothenburg Society of Medicine (GLS), the ALF/LUA grant from the Sahlgrenska University Hospital, and Gustaf V:s och Drottning Victorias Frimurarstiftelse.

\section{Compliance with ethical standard}

Conflicts of interest ADP owns shares of Active Life Scientific, the manufacturer of the OsteoProbe device. Robert Rudäng, Michail Zoulakis, Daniel Sundh, Dan Mellström, Lisa Johansson, Anna Darelid, and Mattias Lorentzon state that they have no conflicts of interest. 
Open Access This article is distributed under the terms of the Creative Commons Attribution-NonCommercial 4.0 International License (http:// creativecommons.org/licenses/by-nc/4.0/), which permits any noncommercial use, distribution, and reproduction in any medium, provided you give appropriate credit to the original author(s) and the source, provide a link to the Creative Commons license, and indicate if changes were made.

\section{References}

1. Kanis JA, Johnell O, Oden A, Sembo I, Redlund-Johnell I, Dawson A, De Laet C, Jonsson B (2000) Long-term risk of osteoporotic fracture in Malmo. Osteoporos Int 11:669-674

2. Kanis JA, Melton LJ 3rd, Christiansen C, Johnston CC, Khaltaev N (1994) The diagnosis of osteoporosis. J Bone Miner Res 9:11371141

3. Stone KL, Seeley DG, Lui LY, Cauley JA, Ensrud K, Browner WS, Nevitt MC, Cummings SR (2003) BMD at multiple sites and risk of fracture of multiple types: long-term results from the Study of Osteoporotic Fractures. J Bone Miner Res 18:1947-1954

4. Kanis JA, Johnell O, Oden A, Johansson H, McCloskey E (2008) FRAX and the assessment of fracture probability in men and women from the UK. Osteoporos Int 19:385-397

5. Kanis JA, Oden A, Johansson H, Borgstrom F, Strom O, McCloskey E (2009) FRAX and its applications to clinical practice. Bone 44:734-743

6. Boutroy S, Van Rietbergen B, Sornay-Rendu E, Munoz F, Bouxsein ML, Delmas PD (2008) Finite element analysis based on in vivo HR-pQCT images of the distal radius is associated with wrist fracture in postmenopausal women. J Bone Miner Res 23: 392-399

7. Kopperdahl DL, Aspelund T, Hoffmann PF, Sigurdsson S, Siggeirsdottir K, Harris TB, Gudnason V, Keaveny TM (2014) Assessment of incident spine and hip fractures in women and men using finite element analysis of CT scans. J Bone Miner Res 29:570-580

8. Diez-Perez A, Guerri R, Nogues X et al (2010) Microindentation for in vivo measurement of bone tissue mechanical properties in humans. J Bone Miner Res 25:1877-1885

9. Guerri-Fernandez RC, Nogues X, Quesada Gomez JM, Torres Del Pliego E, Puig L, Garcia-Giralt N, Yoskovitz G, Mellibovsky L, Hansma PK, Diez-Perez A (2013) Microindentation for in vivo measurement of bone tissue material properties in atypical femoral fracture patients and controls. J Bone Miner Res 28:162-168

10. Bridges D, Randall C, Hansma PK (2012) A new device for performing reference point indentation without a reference probe. Rev Sci Instrum 83:044301

11. Randall C, Bridges D, Guerri R et al (2013) Applications of a new handheld reference point indentation instrument measuring bone material strength. J Med Devices 7:410051-410056

12. Malgo F, Hamdy NA, Papapoulos SE, Appelman-Dijkstra NM (2015) Bone material strength as measured by microindentation in vivo is decreased in patients with fragility fractures independently of bone mineral density. J Clin Endocrinol Metab 100:20392045

13. Farr JN, Drake MT, Amin S, Melton LJ 3rd, McCready LK, Khosla S (2014) In vivo assessment of bone quality in postmenopausal women with type 2 diabetes. J Bone Miner Res 29:787-795
14. Duarte Sosa D, Vilaplana L, Guerr IR, Nogues X, Wang-Fagerland M, Diez-Perez A, Eriksen FE (2015) Are the high hip fracture rates among Norwegian women explained by impaired bone material properties? J Bone Miner Res 30(10):1784-9

15. Genant HK, Li J, Wu CY, Shepherd JA (2000) Vertebral fractures in osteoporosis: a new method for clinical assessment. J Clin Densitom 3:281-290

16. Genant HK, Wu CY, van Kuijk C, Nevitt MC (1993) Vertebral fracture assessment using a semiquantitative technique. J Bone Miner Res 8:1137-1148

17. Hermanussen M, Danker-Hopfe H, Weber GW (2001) Body weight and the shape of the natural distribution of weight, in very large samples of German, Austrian and Norwegian conscripts. Int J Obes Relat Metab Disord 25:1550-1553

18. Portier K, Tolson JK, Roberts SM (2007) Body weight distributions for risk assessment. Risk analysis. Off Publ Soc Risk Anal 27:1126

19. Cheng XG, Lowet G, Boonen S, Nicholson PH, Brys P, Nijs J, Dequeker J (1997) Assessment of the strength of proximal femur in vitro: relationship to femoral bone mineral density and femoral geometry. Bone 20:213-218

20. Farr JN, Tomas R, Chen Z, Lisse JR, Lohman TG, Going SB (2011) Lower trabecular volumetric BMD at metaphyseal regions of weight-bearing bones is associated with prior fracture in young girls. J Bone Miner Res 26:380-387

21. Mellibovsky L, Prieto-Alhambra D, Mellibovsky F, GuerriFernandez R, Nogues X, Randall C, Hansma PK, Diez-Perez A (2015) Bone tissue properties measurement by reference point indentation in glucocorticoid-induced osteoporosis. J Bone Miner Res 30(9):1651-6

22. Darelid A, Ohlsson C, Rudang R, Kindblom JM, Mellstrom D, Lorentzon M (2010) Trabecular volumetric bone mineral density is associated with previous fracture during childhood and adolescence in males: the GOOD study. J Bone Miner Res 25:537-544

23. Nevitt MC, Cummings SR, Browner WS, Seeley DG, Cauley JA, Vogt TM, Black DM (1992) The accuracy of self-report of fractures in elderly women: evidence from a prospective study. Am J Epidemiol 135:490-499

24. Mackey DC, Lui LY, Cawthon PM, Bauer DC, Nevitt MC, Cauley JA, Hillier TA, Lewis CE, Barrett-Connor E, Cummings SR (2007) High-trauma fractures and low bone mineral density in older women and men. Jama 298:2381-2388

25. Sanders KM, Pasco JA, Ugoni AM, Nicholson GC, Seeman E, Martin TJ, Skoric B, Panahi S, Kotowicz MA (1998) The exclusion of high trauma fractures may underestimate the prevalence of bone fragility fractures in the community: the Geelong Osteoporosis Study. J Bone Miner Res 13:1337-1342

26. Hasserius R, Redlund-Johnell I, Mellstrom D, Johansson C, Nilsson BE, Johnell O (2001) Vertebral deformation in urban Swedish men and women: prevalence based on 797 subjects. Acta Orthop Scand 72:273-278

27. O’Neill TW, Felsenberg D, Varlow J, Cooper C, Kanis JA, Silman AJ (1996) The prevalence of vertebral deformity in European men and women: the European Vertebral Osteoporosis Study. J Bone Miner Res 11:1010-1018

28. Kanis JA, Johnell O, De Laet C, Jonsson B, Oden A, Ogelsby AK (2002) International variations in hip fracture probabilities: implications for risk assessment. J Bone Miner Res 17:1237-1244 